Abanico Agroforestal. Enero-Diciembre 2020; 2:1-22. http://dx.doi.org/10.37114/abaagrof/2020.1 Artículo Revisión. Recibido: 16/06/2019. Aceptado: 15/11/2019. Publicado: 01/04/2020.

\title{
Revisión del aceite de orégano spp. en salud y producción animal
}

Review of the use of oregano oil spp. in animal health and production

\section{Loeza-Concha Henry** (D), Salgado-Moreno Socorro² (D), Ávila-Ramos Fidel ${ }^{3}$ (D), Gutiérrez-Leyva Ranferi² $^{(D)}$, Domínguez-Rebolledo Alvaro4 ${ }^{4}$, Ayala-Martínez Maricela $^{5}$, Escalera-Valente Francisco ${ }^{2 *}$}

${ }^{1}$ Colegio de Posgraduados, Campus Campeche, Sihochac, Champotón, Campeche, México. ${ }^{2}$ Unidad Académica de Medicina Veterinaria y Zootecnia, Universidad Autónoma de Nayarit, Nayarit, México. ${ }^{3}$ División de Ciencias de la Vida, Universidad de Guanajuato Irapuato, Guanajuato, México. ${ }^{4}$ Instituto Nacional de Investigaciones Forestales, Agrícolas y Pecuarias-Mocochá, Mérida, México. ${ }^{5}$ Instituto de Ciencias Agropecuarias, Universidad Autónoma del Estado de Hidalgo. México. *Autor de correspondencia: Francisco Escalera Valente. ${ }^{* *}$ Autor responsable: Henry Loeza-Concha. henryloeza_21@yahoo.com, coco_salgado@hotmail.com, ledifar@hotmail.com, granferi@hotmail.com, alvaroedr@gmail.com, ayalamm78@gmail.com, franescalera@hotmail.com.

\section{RESUMEN}

El uso de los aceites esenciales extraído del orégano son relevantes si tomamos en cuenta sus cantidades de timol, flavonoides, taninos, triterpenos y carvacrol contenido, las cuales le dan su capacidad antioxidante, para disminuir la formación de radicales libres. Además, tiene propiedades antibacteriales, antifúngicas, antiparasitarias, antimicrobianas, antivirales, antialérgicas, vasodilatadoras, estrogénicas, antiinflamatorias, espasmolíticas, antitumorales, entre otras. El objetivo de la revisión fue hacer un análisis preliminar del uso del aceite de orégano spp. en la salud y la producción animal. A manera de conclusión se puede decir que el uso de los aceites esenciales de orégano, principalmente de las subespecies vulgare y Lippia, han sido una alternativa como aditivos en la alimentación en la producción de especies de animales domésticos donde se ha podido mejorar la funcionalidad del sistema digestivo en pollos, se ha logrado reducir las emisiones de metano entérico en rumiantes y reduce malos olores en eses de los cerdos.

Palabras clave: orégano, aceite esencial, producción animal

\begin{abstract}
The use of essential oil extracted from oregano is relevant if we take into account the high concentrations of thymol, flavonoids, tannins, triterpenes and carvacrol, which are substances with antioxidant capacity, since they counteract the formation of free radicals, in addition to having antibacterial, antifungal, antiparasitic, antimicrobial, antiviral, antiallergic, vasodilator, estrogenic, anti-inflammatory, spasmolytic, antitumor properties, among others. The objective was to conduct a preliminary analysis of the use of oregano oil spp. in animal health and production. In conclusion, it can be said that the use of essential oils of oregano, mainly of the subspecies vulgare and Lippia, have been an alternative as feed additives in the production of domestic animal species where functionality has been improved of the digestive system in chickens, it has been possible to reduce enteric methane emissions in ruminants and reduce bad odors in pigs.
\end{abstract}

Keywords: oregano, essential oil, animal production. 


\section{INTRODUCCIÓN}

El orégano es una planta que se distribuye ampliamente en el Mediterráneo europeo, Asia y América (Castillo et al., 2017; Zou et al., 2016; Huerta, 1997); en la actualidad se conocen más de 40 especies de las familias Verbenaceae, Lamiaceae, Compositae y Leguminoseae, siendo las más importantes el orégano Origanum vulgare y el orégano mexicano L. graveolens, L. palmeri, L. alba (Castillo et al., 2017; Huerta, 1997). Estas plantas son fáciles de conseguir y por sus características aromáticas es comúnmente empleado como condimento en recetas culinarias (Albado et al., 2001). Por ello el orégano se ubica como una planta de importancia económica; además, sus aceites esenciales son benéficos para la salud humana y pueden ser utilizados como aditivos naturales en los alimentos para los animales de producción (Hyldgaard et al., 2012).

Los aceites de orégano son una mezcla compleja de cientos de compuestos aromáticos volátiles individuales que se derivan de las diversas especies de orégano (Monu et al., 2016). Estos aceites son conocidos por sus propiedades antiinflamatorias, antibacterianas y antioxidativas, debido a su contenido de carvacrol y timol (Govaris et al., 2010); incluso a bajas concentraciones, el aceite de orégano tiene actividad biológica (Ali et al., 2015). Se ha demostrado que tienen efecto inhibidor sobre una variedad de bacterias y tiene un amplio espectro de propiedades antibacterianas (Gonçalves et al., 2013). En este sentido la propiedad natural del aceite de orégano ha permitido que se pueda utilizar para conservar alimentos (Viuda et al., 2011), como acaricida para el control de parásitos, como la Varroa en las abejas (Loeza et al., 2018).

El uso de aceites esenciales del orégano toma mayor relevancia si consideramos la prohibición de los antibióticos promotores del crecimiento, estimulando la búsqueda de suplementos alimenticios alternativos en la producción de diversas especies de animales (Botsoglou et al., 2002). Por lo tanto, el objetivo de la investigación, fue realizar un análisis preliminar del uso del aceite de orégano spp. en la salud y producción animal.

En los últimos años, los aceites esenciales han tenido impacto en la producción animal, debido a que han demostrado ser una buena alternativa para reducir el uso de compuestos químicos (Martínez et al., 2015); por ello, se ha utilizado en pollos de engorda, con la finalidad de mejorar la digestibilidad ileal; además, estimulan el apetito de las aves y aumenta la ganancia de su peso (Isabel y Santos, 2009). En pavos ha logrado incrementar la estabilidad de su carne cruda y cocida, así como a la oxidación lipídica (Botsoglou et al., 2003). En cerdos reduce la emisión de gases, de coliformes fecales y bacterias anaeróbicas (Varel, 2002); además, en cerdas gestantes reduce la mortalidad de sus lechones (Allan y Bilkei, 2005). En abejas se ha utilizado para el control alternativo de Varroa destructor (Itzá et al., 2007). En rumiantes la presencia de carvacrol, $\rho$-cimeno, linalol, terpineno y timol, lo han utilizado para disminuir la emisiones de metano en el rumen de ovejas, cabras y bovinos (Talebzadeh et al., 2012); y en peces el uso de 
aceite esencial de orégano durante el transporte redujo el estrés oxidativo en los tejidos de los peces (Azambuja et al., 2011).

A pesar de los beneficios obtenidos de las diferentes especies de orégano en la producción animal, han logrado la prevención de enfermedades, la respuesta del sistema inmune de los animales y la productividad de los diversos sistemas productivos (Martínez et al., 2015). Se ha dejado a un lado su funcionalidad en la reproducción de diversas especies, esto se puede fundamentar principalmente por las altas cantidades de antioxidante presentes en los aceites esenciales del orégano. Pueden mejorar la calidad seminal del semen fresco y la resistencia de los espermatozoides al shock térmico durante el proceso de criopreservación (Alvarez y Storey, 1992).

La inclusión del timol extraído de las plantas de orégano a las dietas de las abejas inoculadas con Nosema, ha logrado reducir la infección (Van den et al., 2016; Costa et al., 2010; Maistrello et al., 2008). Sin embargo, estas investigaciones se realizaron en laboratorio, controlando la ingesta del timol. No se conoce su eficiencia en campo o en condiciones naturales; por lo tanto, se deben tener precausiones al usar timol para la erradicación de Nosema, hasta encontrar las dosis apropiadas y el método de aplicación correcto. Los beneficios dependen de la especie de orégano utilizado o la combinación de aceites esenciales.

Barreto et al. (2008) indican que el efecto del aceite esencial de orégano se puede potencializar adicionando aceites esenciales de otras plantas; sin embargo, Hernandez et al. (2004) lo combinaron con canela y pimienta; pero no aumentó la ganancia de peso o conversión alimenticia en aves. Oetting et al. (2006) lo mezclaron con tomillo y clavo en dietas para lechones de 28 días, pero los animales bajaron de peso y la conversión alimenticia fue mayor, comparada con el antibiótico.

En la actualidad, muchos beneficios de los aceites esenciales del orégano están descritos, pero no se conocen los efectos adversos al usarlo indiscriminadamente; por lo tanto, es necesaria la implementación y generación de nuevos conocimientos que puedan describir a la planta y cómo se puede usar.

\section{REVISIÓN DE LITERATURA}

\section{La planta de orégano}

El nombre del género Oreganum proviene del vocablo griego, oros que significa montaña y ganos, alegría; en referencia al bello aspecto que esta planta confiere a las regiones donde crece. El orégano es originario de Asia y Europa, pero se cultiva en regiones templadas de varios países. Las plantas también son cultivadas por sus propiedades terapéuticas, farmacéuticas apícolas y en la herboristería; lo consumen ampliamente por sus propiedades tónicas, digestivas, estomacales y antiasmáticas (INFOAGRO, 2006). 


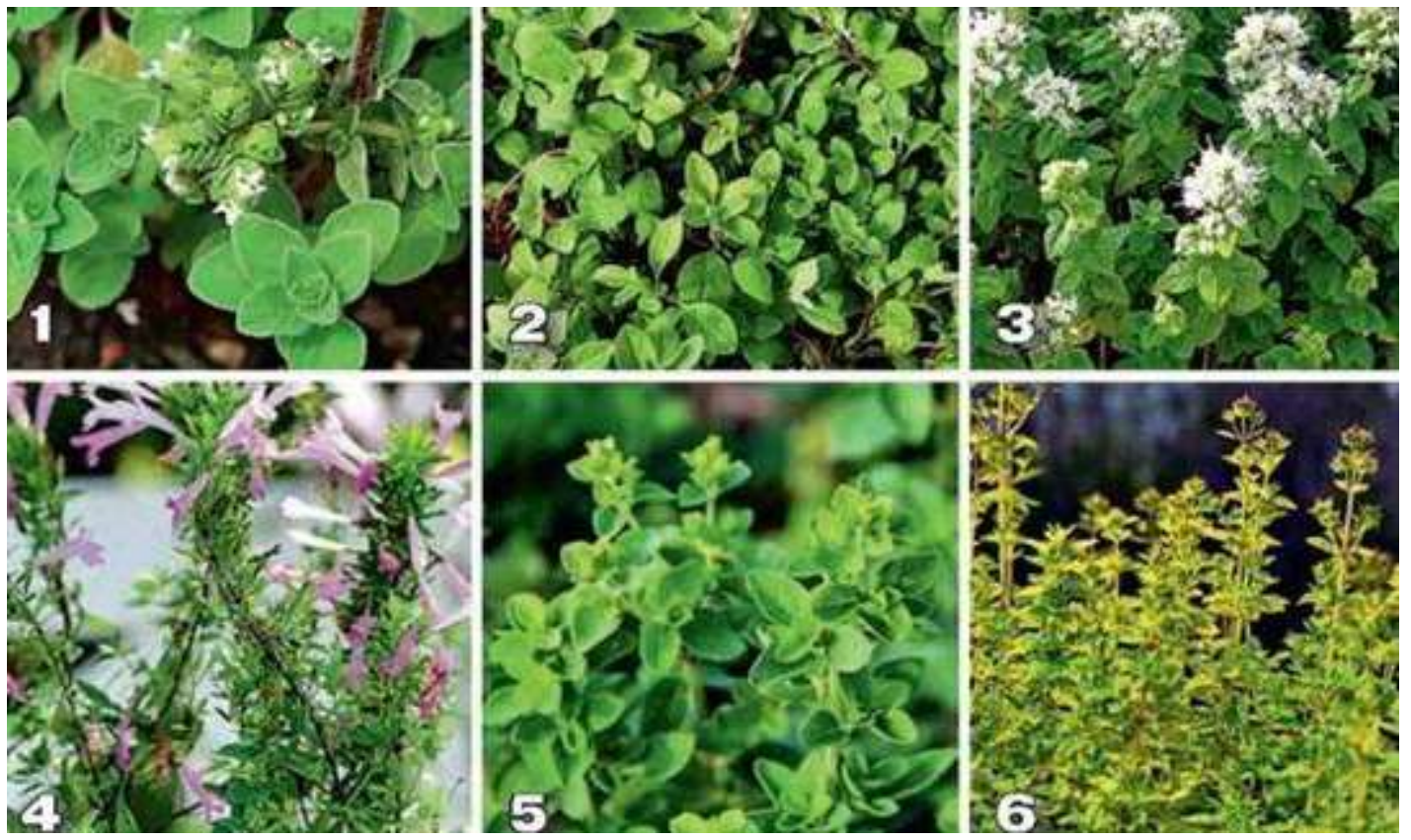

Imagen 1. Variedades de orégano.

1: orégano italiano (Origanum majoricum); 2: orégano del mediterráneo oriental, (Origanum majorana); 3 : orégano turco (Origanum onites); 4: orégano mexicano (Poliomintha longrflora); 5: orégano griego (Origanum heracleoticum); 6: orégano de la Península Ibérica y Baleares (Origanum vulgare). Fuente: http://vsaduidoma.com/es/2016/06/27/dushica-i-majoran-foto-otlichiya-i-raznost-v-posadke-i-uhode/

\section{Especies de orégano}

El orégano pertenece a la familia Lamiaceae plata, especialmente aromática; su taxonomía se describe en la tabla 1 (WCSP, 2014). En la actualidad se han reportado 61 especies de orégano, contenidas en 17 géneros de seis familias bajo este nombre. El género Origanum (familia Labiatae), conocido como orégano europeo, se considera el más importante (tabla 2); sin embargo, en el continente americano los géneros Lanata y Lippia (familia Verbenaceae) son los oréganos mexicanos más abundantes; pero existen otras familias (Rubiaceae, Scrophulariaceae, Apiaceae y Asteraceae) que no tienen impacto productivo (WCSP).

\section{Descripción botánica}

Las diversas variedades de orégano son plantas herbáceas, perennes en forma de un pequeño arbusto achaparrado, de unos $45 \mathrm{~cm}$ a $60 \mathrm{~cm}$ de altura; toda la planta está cubierta de pelos glandulares; su tallo adquiere una tonalidad rojiza, se ramifican en la parte superior y tienden a deshojarse en las partes inferiores. Las hojas superiores son más pequeñas que las inferiores; las hojas opuestas en las márgenes tienen glándulas ciliadas llenas de aceites esenciales. Las flores son de color rosa, purpura o violeta, dependiendo de la especie, y los frutos generalmente son tetraquenios y secos (Fonnegra, 2007). 
Tabla 1. Clasificación taxonómica del Orégano

\begin{tabular}{cc}
\hline Reino: & Plantae \\
División: & Magnoliophyta \\
Clase: & Magnoliopsida \\
Orden: & Lamiales \\
Familia: & Lamiaceae \\
Subfamilia: & Nepetoideae \\
Tribu: & Mentheae \\
Género: & Origanum \\
Especie: & O. vulgare \\
Nombre científico: & Origanum vulgare L \\
Nombre común: & Orégano \\
\hline & Fuente: National Library of Medicine
\end{tabular}

Tabla 2. Especies de orégano utilizados a nivel mundial

\begin{tabular}{|c|c|c|}
\hline - Labiatae & $\begin{array}{l}\text { - Hirtum } \\
\text { Virens } \\
\text { - Viridulum }\end{array}$ & 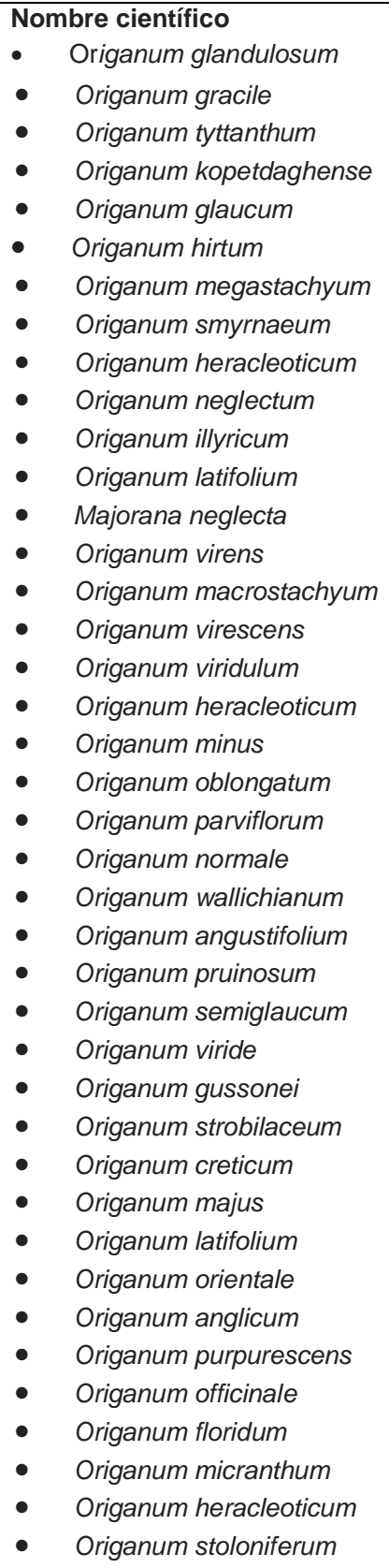 \\
\hline
\end{tabular}


- Verbenácea

- Lanata

- $\quad$ Lippia
- Rubiaceae

- Scrophulariaceae

- Apiaceae

- Asteraceae
- $\quad$ Origanum thymiflorum

- Origanum laxiflorum

- Origanum loureiroi

- Origanum decipiens

- Origanum americanum

- Origanum capitatum

- Origanum nutans

- Origanum venosum

- Oroga heracleotica

- Origanum serpylliforme

- Origanum albiflorum

- Origanum megastachyum

- Origanum watsonii

- Origanum barcense

- Origanum elegans

- Micromeria formosana

- Origanum dilatatum

- Origanum puberulum

- Mentha formosana

- Lantana citrosa

- Lantana glandulosissima

- Lantana involucrata

- Lantana purpurea

- Lantana trifolia

- Lantana velutina

- Lippia myriocephala

- Lippia affinis

- Lippia alba

- Lippia berlandieri

- Lippia cardiostegia

- Lippia formosa

- Lippia geisseana

- Lippia graveolens

- Lippia helleri

- Lippia micromera

- Lippia micromera

- Lippia origanoides

- Lippia palmeri

- Lippia palmeri

- Borreria sp.

- Limnophila stolonifera

- Eryngium foetidum $L$

- Coeosanthus veronicaefolius

- Eupatorium macrophyllum L.

\section{Producción de orégano en México}

El orégano mexicano es una fuente importante de ingresos para las poblaciones marginadas del país en expansión, debido a su demanda (Cazares-Alonso et al., 2010). El 90\% de la producción se encuentra distribuida de manera silvestre en 24 estados de la República Mexicana, con una producción anual de 4,000 toneladas (García-Pérez et 
al., 2012; Casillas-Alcalá, 1992). En los últimos años se han instalado cultivos comerciales en los Estados de Durango, Guanajuato, Jalisco, Querétaro, San Luis Potosí Coahuila, Tamaulipas, Nuevo León y Zacatecas (García et al., 2012).

Las exportaciones del orégano mexicano están destinadas al Reino Unido, Alemania, Francia y Canadá. En los últimos años se ha registrado que las ventas del orégano mexicano han aumentado a los 2 millones de dólares (CONAFOR, 2009). El incremento de su precio se atribuye a la demanda tanto nacional como internacional, estimulando el desarrollo de tecnología para el establecimiento de cultivos más eficientes (CazaresAlonso et al., 2010).

Los géneros de orégano más cultivados en México, destacan la Lantana y la Lippia (con tres y dos especies, respectivamente); esto es debido a que son los más explotados en nuestro pais; posiblemente por que se encuentran en gran parte del territorio nacional de manera silvestre, en regiones áridas y semiáridas de México (García et al., 2012). Sin embargo, a pesar de su importancia económica, su explotación no está incluida en los programas básicos de manejo y mejoramiento agronómico, puesto que la producción comercial del orégano mexicano demanda homogeneidad, volumen y calidad; factores que se contraponen al tipo de colecta, ya que ésta se realiza en zonas marginadas y de escasos ingresos, generando una explotación desmedida que ponen en peligro la biodiversidad y sustentabilidad de la misma.

Por estas razones es necesario asegurar un manejo racional de este recurso, para poder impactar positivamente el nivel socioeconómico de las familias en las regiones donde se produce (Huerta, 1997). Debido a las necesidades mencionadas anteriormente, se han establecido las bases para el manejo agronómico del orégano, destacando la recolecta de semillas antes de la cosecha para renovar las poblaciones. Se ha propuesto su propagación por medio de estacas, utilizando ácido indol-butírico (2000ppm) como enraizador. Se ha sugerido que el corte de la planta se realice hasta que alcance su madurez y después de la floración. La calidad de la planta cultivada bajo este esquema es óptima para su explotación hasta los 3 años, y finalmente se ha determinado que la mejor época para plantar orégano, en condiciones agro-climáticas de sierra, es entre los meses de septiembre a diciembre, época que presenta temperaturas favorables (Corella y Ortega, 2013).

\section{Valor comercial del orégano}

La producción mundial de orégano genera un valor aproximado de $\$ 22.5$ millones de dólares; no obstante, la Comisión Nacional Forestal estimó que en 2005 las ventas totales de orégano sumaron más de $\$ 75$ millones de euros (Koksal et al., 2010). En México la producción de orégano representa una derrama económica de 5.6 millones de pesos, haciendo que esta actividad sea de impacto para el sector rural y una fuente de empleo en áreas de alta marginación. 
México se encuentra ubicado como el segundo país productor de orégano a nivel mundial, aporta entre del $35 \%$ al $40 \%$ de la producción total a nivel mundial (Soto et al., 2007; García et al., 2012). El 85\% de la producción es exportada a los EE.UU y el 5\% a países Europeos y Asiáticos (Castillo et al., 2017). De las 5 especies de oréganos comerciales en México, las especies Lippia graveolens H.B.K. y Lippia Berlandieri Schauer son los oréganos de mayor explotación e importancia económica, debido a que estas dos especies de orégano están desplazando a productos que provienen de Grecia y Turquía. El orégano mexicano contiene mejor composición química de sus aceites esenciales, lo que le ha permitido una mayor comercialización en los últimos años. El costo promedio de la hoja seca de orégano por kg varía de 8 a 11 pesos mexicanos (Nieves et al., 2010).

\section{Composición química}

El aceite esencial de orégano es rico en: timol, beta- bisaboleno, cariofileno, p-cimeno, borneol, lianalol, ecetato de linalilo, alfa y beta-pinenos, alfa-terpineno, ácidos fenolcaboxílico, como: caféico, clorogénico y rosmarínico. Contiene flavonoides, como: derivados de apigenol, luteolol, kenferol y diosmetol. También contiene algunos triterpenos, como: derivado de los ácidos ursólicos y olenaolicos; además de taninos (Fonnegra, 2007).

\section{Valor nutricional del orégano}

El orégano es utilizado en la alimentación de humanos y como aditivo en las dietas de los animales, debido a las proteínas, hierro, calcio, potasio, magnesio, zinc, fósforo, niacina, vitamina A, timol y carvacrol (Tabla 3) (Moreiras et al., 2013).

\section{Propiedades medicinales del aceite esencial de orégano}

El valor comercial del orégano se debe a sus características, como especia, condimento y propiedades medicinales; sin embargo, la mayor importancia industrial y farmacéutica de la planta es su aceite esencial empleado como fragancia en jabones, perfumes, cosméticos y saborizantes, principalmente (Koksal et al., 2010). Además, el aceite de orégano contiene flavonoides, sustancias importantes en el área farmacológica, por su capacidad antioxidante en la formación de radicales libres, cuya influencia posee propiedades: antibacteriales, antifúngicas, antiparasitarias, antimicrobianas, antioxidantes, antivirales, antialérgicas, vasodilatadores, antiinflamatorias, antimicrobianas, entre otras (Cáceres et al., 2014; Soto et al., 2012; Meneses et al., 2009, Gonzalez et al; 2009; Güereca et al., 2007; García et al., 2006). 
Tabla 3. Composición nutricional por cada 100 gr de orégano seco

\begin{tabular}{crr}
\hline Composición & Cantidad (gr) & \multicolumn{2}{c}{ CDR (\%) } \\
\hline K calorías & 308 & $16.1 \%$ \\
Carbohidratos & 21.63 & $7 \%$ \\
Proteínas & 11 & $23 \%$ \\
Fibra & 42.8 & $142.7 \%$ \\
Grasas & 10.25 & $19.3 \%$ \\
Sodio & 15 & $0.9 \%$ \\
Sodio & 15 & $0.9 \%$ \\
Calcio & 1576 & $131.3 \%$ \\
Hierro & 44 & $550 \%$ \\
Magnesio & 0 & $0 \%$ \\
Fósforo & 200 & $28.6 \%$ \\
Potasio & 1669 & $83.5 \%$ \\
Vitamina A & 0.69 & $76.7 \%$ \\
Vitamina B1 & 0.34 & $28.3 \%$ \\
Vitamina B2 & 0.32 & $24.6 \%$ \\
Vitamina B3 & 6.22 & $0 \%$ \\
Vitamina B12 & 0 & $0 \%$ \\
Vitamina C & 50 & $55.6 \%$ \\
\hline & \multicolumn{2}{c}{ Fuente: Moreiras et al. 2013}
\end{tabular}

\section{Actividad antioxidante}

En la Actualidad se han realizado estudios sobre la actividad antioxidante del orégano, donde autores como Soto et al. (2012) indican que el orégano presenta actividad antioxidante, la cual incrementa conforme aumentan las concentraciones del extracto sin tener un efecto tóxico in vitro e in vivo. Las concentraciones de antioxidantes entre plantas varía, debido a las diferencias en la composición y cantidad de los metabolitos secundarios. Pero hay factores como la zona geográfica, clima, altitud, época de cosecha y su estado de crecimiento que afectan su contenido (Güereca et al., 2007). Muchas especias y hierbas de la familia Lamiaceae a la que pertenece el orégano, han sido evaluadas como antioxidantes y conservadores en alimentos, debido a que se ha demostrado que el orégano logra conservar a bajas temperaturas diversos alimentos, pues se conoce que el timol y el carvacrol actúan como antioxidantes de lípidos (Yanishlieva et al., 1999); es decir, que su actividad antioxidante está asociada a varios mecanismos; además que su elevada reactividad frente a radicales libres activos se considera el mecanismo principal (Cervato et al., 2000).

La importancia del orégano en la industria alimentaria se ha visto incrementada por su uso como aditivo alternativo para la conservación de los alimentos (Dorman et al., 2003). Se ha demostrado que las sustancias responsables de la actividad antioxidante del orégano son los compuestos fenólicos por su estructura molecular, en especial, el grado de hidroxilación y la posición de los oxhidrilos que éstas contienen (Kulisic et al., 2004). Además sus principios activos pueden actuar en una o varias de las etapas de la secuencia oxidativa, constituyendo una de las principales clases de metabolitos secundarios de las plantas, las cuales desempeñan diversas funciones fisiológicas (Gotsiou et al., 2002). El orégano puede llegar a inhibir la oxidación de biomoléculas (proteínas y DNA), impidiendo la iniciación o propagación de las especies reactivas de oxígeno (ERO), las cuales están relacionadas con la incidencia de varias patologías 
humanas, entre ellas: cáncer, cardiopatías, problemas neurodegenerativos, como Alzheimer, Parkinson; además de procesos de envejecimiento (Aiyegoro y Okoh, 2009).

\section{Actividad Antimicrobiana}

Existen múltiples estudios sobre la actividad antimicrobiana de los extractos de diferentes tipos de aceite de orégano; presentan actividad contra bacterias gram negativas, como: Salmonella typhimurium, Escherichia coli, Klebsiella pneumoniae, Yersinia enterocolitica y Enterobacter cloacae; y las gram positivas, como: Staphylococcus aureus, Staphylococcus epidermidis, Listeria monocytogenes y Bacillus subtilis (Elgayyar et al., 2001). Los aceites esenciales obtenidos de las especies de oréganos contienen un potencial poder antimicrobiano, puesto que se han evaluado y se ha demostrado que son efectivas contra varios microorganismos diferentes (Arcila et al., 2004).

La efectividad del aceite de orégano como antimicrobiano se atribuye a dos compuestos presentes: carvacrol y timol; los cuales inhiben a los microorganismos patógenos, ya que impiden el desarrollo de la actividad microbiana de microorganismos gram negativos, (Santoyo et al., 2006; Yano et al., 2006). La actividad antimicrobiana depende de la composición química del aceite esencial de orégano, la cual está relacionada con la especie de orégano, condiciones geográficas, periodos de cosecha, método de extracción y las concentraciones mínimas inhibitorias (CMI), las cuales se han establecido entre $0.28-1.27 \mathrm{mg} / \mathrm{ml}$ para las bacterias (Hazzit et al., 2006; Aligiannis et al., 2001).

\section{Actividad antifúngica}

En estudios recientes el aceite esencial de orégano en concentración de $0.3 \%, 0.5 \%$, $0.7 \%$ y $1.0 \%$ presentaron una inhibición del crecimiento micelial al $100 \%$, frente a los hongos fitopatógenos aislados, y se ha demostrado que tiene capacidad antifúngica contra: Cándida albicans, C. tropicalis, Torulopsis glabrata, Aspergillus Níger, Geotrichum y Rhodotorula; Botrytis cinerea, Rhizopus stolonifer, Colletotrichum sp., Penicillium italicum, Penicillium digitatum, Fusarium moniliforme, Aspergillus flavus, Aspergillus parasiticus, Fusarium graminearum, Aspergillus ochraceus, entre otros. Se ha informado del efecto inhibitorio del aceite esencial de orégano sobre el desarrollo de diversos hongos in vitro; además se determinó que con 2000 ppm de aceite de orégano se puede controlar el crecimiento micelial de estos hongos, así mismo, se logró demostrar que el aceite esencial de orégano controla eficientemente in vivo el desarrollo de hongos endógenos en trigo (Cáceres et al., 2014; García et al., 2006); dichas capacidades se atribuyen a la actividad fungicida de los aceites esenciales de orégano, especialmente a la presencia de timol y carvacrol en dichas sustancias (Madsen y Bertelsen, 1995). 


\section{Actividad antiviral}

Los extractos de orégano han sido estudiados por las propiedades de sus aceites esenciales, sobre la actividad infectiva del virus de la fiebre amarilla (Meneses et al., 2009). Los aceites esenciales de orégano fueron eficientes en la inhibición de cinco virus de ADN (HHV-1, ACVR-HHV-1, BoHV-1, BoHV-2, BoHV-5) y tres virus de ARN (HRSV, $R V, B V D V)$. Este efecto inhibitorio se le atribuye principalmente al método de extracción del aceite y la parte de la planta seleccionada (Meneses et al., 2009). Por el contrario, en otro estudio realizado por García-Pérez et al. (2012), se determinó ineficiencia antiviral sobre la reproducción del virus de la influenza A/Aichi/2/68 (H3N2) en células MDCK.

\section{Actividad anti-inflamatoria}

Algunos de los fitoquímicos solubles han sido recientemente re-evaluados en su efecto anti-inflamatorio. Se ha reportado que el extracto de orégano soluble en agua inhibe la secreción de la ciclooxigenasa 2 (COX-2), mostrando una actividad anti-inflamatoria en células humanas de carcinoma epitelial. Asimismo, un extracto etanólico de orégano exhibió la actividad anti-inflamatoria en un modelo de ratón con gastritis, inducida por estrés e hipersensibilidad por contacto. Los principales fitoquímicos responsables de la actividad anti-inflamatoria son el ácido rosmarínico, el ácido ursólico y al ácido oleanólico (Peralta, 2018; García et al., 2012). De la misma manera, se ha determinado la presencia de los flavonoides kampferol, isokampférido y pilosina; los cuales tienen una actividad biológica antiinflamatoria, antiulcerogénica y vasoconstrictora, que justifican su uso medicinal, como antiespasmódica, analgésica, antiinflamatoria y antihemorrágica (Güereca et al., 2007).

\section{Uso del aceite esencial de orégano en diferentes especies animales}

Actualmente existe la tendencia mundial por sustituir los productos químicos sintéticos, por el uso de aceites esenciales con actividad antiparasitaria, antiviral, antifúngica y antibacteriana (Meneses et al., 2009; Santoyo et al., 2006; Yano et al., 2006); debido a las preocupaciones públicas y políticas, relacionadas con el uso elevado de productos sintéticos, los cuales presentan potenciales riesgos de causar resistencia a los diversos patógenos presentes en los animales de consumo humano, lo que compromete la producción mundial de alimentos proteicos (carne, leche, huevo) y la miel (Cabrera et al., 2007).

\section{Aves}

Los compuestos de los aceites esenciales de orégano son una alternativa de reemplazo de los antibióticos promotores de crecimiento, permitiendo potenciar el desempeño funcional integral del sistema digestivo y la expresión productiva del pollo de engorde; esto es debido a que la inclusión de extractos de orégano en la dieta, mejora la digestibilidad ileal y total de materia seca, extracto etéreo y del almidón (Betancourt, 2012). Estos efectos funcionales han sido atribuidos al contenido de los fenoles; carvacrol 
y timol, en rangos que van desde $3 \%$ hasta $75 \%$ del total del aceite; con la presencia de otros componentes como monoterpenos hidrocarbonados, $y$-terpineno y pcimeno (Aligiannis et al., 2001).

El aceite esencial de orégano, también ha sido utilizado como aditivo en las dietas de aves destinadas a la producción de carne, esto es debido a que el aceite de orégano tiene efectos benéficos sobre la actividad antibacteriana del tracto intestinal de pollos de engorda, lo que ha beneficiado a la producción de las explotaciones avícolas; debido a que la adición de aceite esencial de orégano ha mejorado la calidad de la canal (Betancourt, 2012); asimismo, en pollos de engorda se ha demostrado que una mezcla de aceites esenciales de clavo (Syzigium aromathicum) y orégano (Origanum vulgare) estimulan el apetito de las aves, y de esta manera se mejora la conversión; es decir, que la inclusión de aceites esenciales a la dieta de pollos logra aumentar la ganancia de peso, mejorar la conversión alimenticia y en general los parámetros productivos (Isabel y Santos, 2009). Cómo antioxidante, el orégano fue ensayado en carne de pavo, y los resultados mostraron que en concentración de $200 \mathrm{mg} / \mathrm{kg}-1$ de alimento aumentó la estabilidad de la carne cruda y cocida a la oxidación lipídica, en comparación con el grupo que no contenía aceites esenciales (Botsoglou et al., 2003).

Se ha demostrado que el uso de aceite esencial de orégano con $27.67 \%$ de timol + $11.31 \%$ de carvacrol logró inhibir la presencia de mesófilos aerobios y patógenos ( $S$. typhy, S. aureus y E. coli) en carne de pavos (Gonzales et al., 2009); del mismo modo Domínguez et al. (2015), demostraron que el uso de $400 \mathrm{mg}$ de aceite de orégano por $\mathrm{kg}$ de alimento de pollos de engorda, disminuyó la cantidad de mesófilos aerobios en carne fresca y congelada de pollos de engorda de 35 y 42 días de edad (el aceite de orégano contenía $43.17 \%$ y $29.16 \%$ de timol y carvacrol, respectivamente). De acuerdo a lo anterior, el uso de aceite esencial de orégano es una alternativa viable en las dietas de aves, debido que la ingesta de alimento, ganancia de peso, índice de conversión alimenticia y mortalidad no se ven afectadas cuando se hace uso de dicho aceite esencial (Fonseca et al., 2017); además de que la mortalidad disminuye con la inclusión de aceite de orégano en la dieta de las aves (Escalera et al., 2016).

\section{Cerdos}

En los cerdos, se ha encontrado que la utilización de $2.5 \mathrm{~g}$ de carvacrol o timol por litro de excretas porcinas, inhibe completamente la producción de los compuestos que le dan mal olor, como: valerato, isobutirato, cresol y isovalerato; reduciendo la emisión de gases, de coliformes fecales y bacterias anaeróbicas (Varel, 2002). En otro estudio, la inclusión del aceite esencial de orégano en las dietas de cerdas en preparto y lactancia, presentaron menor tasa de mortalidad, mayor tasa de nacimientos, más lechones nacidos vivos, menos peso bajo de lechones al nacimiento y un mayor consumo voluntario de alimento (Allan y Bilkei, 2005). Asimismo, Ariza et al. (2011) informaron que la inclusión del orégano a las dietas de las cerdas incrementó la tasa de crecimiento en los lechones, 
lo que coincide con los resultados obtenidos por Guerra et al. (2008), donde demostraron que el aceite de orégano produce mejores efectos en ganancia de peso y peso final, y con los resultados obtenidos por Khajarern y Khajarern (2002); donde también informaron que cuando se agregó aceite esencial de orégano a la dieta, se observó un aumento en la ingesta diaria de alimento de las cerdas lactantes y el aumento de peso diario de sus crías, en comparación con las crías de cerdas no alimentadas con aceite de orégano; además que el uso de orégano disminuye el tiempo de permanencia del alimento en su tránsito por el tracto gastrointestinal, puesto que se comprobó que una mezcla de extractos de plantas, modifica la tasa de vaciado del estómago en cerdos destetados. Posiblemente estos efectos soportan y explican la mejor digestibilidad ideal obtenida por Betancourt (2012), con la inclusión de aceite de orégano.

\section{Abejas}

En la apicultura se ha hecho uso de sustancias orgánicas, como el aceite esencial de orégano; ésta ha sido utilizada para el control alternativo de Varroa destructor y Nosema, debido a su eficacia, fácil aplicación y el bajo riesgo de contaminación de la miel y cera producida en las colonias, cuando estas son sometidas a tratamiento; además que los ácaros no presentan resistencia a este producto alternativo (Romo et al., 2016; Itzá et al., 2007). En la apicultura mundial, en la última década se está haciendo uso del timol, el cual es obtenido principalmente de las plantas de orégano; en este sentido el timol es uno de los productos naturales más utilizados para el control de la varroasis; sin embargo, su eficacia varía de acuerdo con las condiciones climáticas de cada región, tiempo de aplicación, concentración, y forma de aplicación (en gel, polvo o aceite). Itzá et al. (2007), reportaron que la eficacia del timol puede variar entre un $66 \%$ a un $98 \%$; del mismo modo Romo et al., (2016) sugieren que el aceite de orégano puro es una alternativa viable para controlar Varroa en abejas melíferas; además que la cantidad de carvacrol encontrada en la miel producida durante la aplicación del tratamiento a base de aceite esencial de orégano, no excedió el umbral de detección de sabor de $0.1 \mathrm{ppm}$, por lo que se puede considerar como una alternativa viable, económica y sin ningún impacto ambiental.

La importancia del uso del timol obtenido del orégano, radica también en los efectos dañinos que éste le causa a los ácaros de Varroa, ya que en un estudio realizado por Loeza et al. (2018) lograron observar que cuando se usa el timol en una concentración del $20 \%$ durante 28 días, los ácaros de Varroa presentan una plasticidad; por lo que se encontró una reducción significativa en el ancho del escudo dorsal y en largo del escudo genital de la Varroa; sin embargo, se sugiere realizar más estudios, ya que la reducción del largo del escudo genital pudiera tener efectos adversos en la reproducción de los ácaros, lo que pudiera ser benéfico para los apicultores, ya que de esta manera se pudiera reducir la ovoposición de las hembras de Varroa, disminuyendo así los niveles de infestación de las colmenas.

El uso de timol incrementa su importancia en la erradicación de patógenos, por lo que el efecto del timol puede reducir tanto la presencia de Varroa como la de Nosema, hecho 
que ha sido comprobado por Rice (2001) y Maistrello et al., (2008); quienes indican que el timol actúa penetrando las capas de las esporas de Nosema, impidiendo la germinación y el desarrollo de la enfermedad causada por la replicación del esporoplasma, lo que permite controlar esta enfermedad. En consideración a lo antes mencionado, el aceite esencia de orégano y sus componentes, principalmente el timol, son una solución ante los riesgos que causan los productos químicos para el control de Varroa y Nosema en las abejas.

\section{Rumiantes (ovejas, cabras y bovinos)}

En rumiantes, el aceite de orégano se ha utilizado para disminuir la emisiones de metano (Talebzadeh et al., 2012), donde autores como Benchaar y Greathead (2011) encontraron que el aceite de orégano en dosis altas $\left(>300 \mathrm{mg}^{-1}\right.$ de fluido de cultivo de líquido ruminal de bovinos) reduce la población microbiana (bacterias metanogénicas, hongos, protozoarios, etc.); lo que es debido a la reducción in vitro de la producción de amoniaco, biomasa microbiana y degradabilidad; además que el aceite (500 mg $\cdot 1-1)$, no inhiben la fermentación microbiana ruminal; sin embargo, sí la modifica, aumentando la concentración de ácidos grasos volátiles y de nitrógeno amoniacal (Geraci et al., 2012).

Newbold (2004) y Benchaar (2007) mencionaron que los aceites esenciales inhiben a las bacterias generadoras de nitrógeno; por tanto, la diseminación de las proteínas decrece, por lo que se ha reportado hasta un $25 \%$ de reducción de estas bacterias cuando se utiliza aceite de orégano de entre 30 y 300 mg; asimismo, Hristov et al. (2013), encontraron de forma in vivo que la inclusión de aceite esencial de orégano a niveles de $250 \mathrm{~g} / \mathrm{d}, 500 \mathrm{~g} / \mathrm{d}$ y $750 \mathrm{~g} / \mathrm{d}$ disminuyen la concentración de amoniaco de los rumiantes. En este contexto los principales químicos del orégano, son el carvacrol, $\rho$-cimeno, linalol, terpineno y timol; los cuales presentan una alta capacidad antioxidante y su potencial antimicrobiano, que pueden afectar el desarrollo y crecimiento de las bacterias ruminales e inhibir la metanogénesis; además que la inclusión de $300 \mathrm{mg} / \mathrm{L}$ y de $3000 \mathrm{mg} / \mathrm{L}$ de aceite esencial de orégano ayuda a reducir los ácidos grasos volátiles totales.

El aceite esencial de orégano se debe utilizar en dosis bajas para poder observar efectos benéficos en la digestibilidad de fibra detergente neutra; se ha observado una mejora en la disponibilidad de energía para los microorganismos ruminales, en comparación con el uso de dosis altas, las cuales causan efectos deletéreos en la digestibilidad de la fibra por su efecto antimicrobiano; es decir, que en las dosis más altas se encontraron efectos negativos sobre la digestibilidad de la materia seca y la fibra (Klevenhusen et al., 2011). Sin embargo, debido a que el aceite esencial de orégano presenta mayores beneficios que desventajas, el aceite esencial de orégano ha sido considerado como una opción con fines de mitigar las emisiones de metano entérico y mejorar la fermentación ruminal con la producción de ácidos grasos volátiles (Durmic et al., 2014; Busquet et al., 2006). 


\section{Peces}

El uso de aceites esenciales ha adquirido gran importancia por los beneficios en la alimentación, el transporte, manejo y salud de los peces; esto es debido a que los aceites esenciales de orégano reducen los niveles de estrés, lesiones, mortalidad y enfermedades; por lo que se ha demostrado que su uso mejora la productividad de los sistemas acuícolas (Abdollahzadeh et al., 2014). En este sentido, el uso de altas cantidades de carvacrol que contiene el aceite esencial de orégano, es una alternativa viable como un complemento de los antibióticos comerciales para el control de Vibrio spp., en camarones peneidos (García et al., 2012), del mismo modo, se ha demostrado que el uso del aceite esencial de orégano en dosis $6 \%, 2 \%$ y $4 \%$ logra inhibir las bacterias Salmonella sp, Proteus sp y Staphylococcus aureus presentes en peces silvestres enfermos (Martínez et al., 2018), es decir que el aceite esencial de orégano es una alternativa para el control de diversos patógenos que pudieran afectar la buena salud de los peces.

El aceite esencial de orégano en la acuicultura es usado como antioxidantes, esto ha sido posible debido a que el orégano constituye una fuente generosa de antioxidantes, debido a las altas concentraciones de timol y de carvacrol presentes de manera natural en las plantas, debido a lo anterior, el aceite esencial de orégano se considera como una opción económica, plausible, inofensiva y efectiva; debido a ello los aceites esenciales de orégano son utilizados como sustancias terapéuticas en la acuicultura (Aanyu et al., 2018); además que proporcionan una solución para el crecimiento deficiente y las respuestas al estrés relacionadas con las condiciones de cultivo intensivo, así como las deficiencias inmunitarias relacionadas con el estrés oxidativo (Knight, 2010), así como promotores de crecimiento dentro de los sistemas de producción de alevinos en fase de masculinización; ya que se ha demostrado que el uso del aceite esencial de orégano en concentraciones del 1\%, incrementa la rentabilidad de los sistemas de producción de tilapia roja (Oreochromis spp) (Coronado, 2019).

El uso del aceite esencial de orégano puede reducir los daños que se producen en los peces durante el transporte y manejo al mercado, o a lugares dentro de la granja; estos beneficios son posibles debido a que el aceite esencial de orégano (Lippia alba), cuando se aplica en una concentración de $10 \mu \mathrm{l} \cdot \mathrm{I}-1$ en peces durante su transporte $(5-7 \mathrm{~h})$, reduce el estrés oxidativo en los tejidos de los peces (hígado, cerebro y branquias); además se ha reportado que una concentración de $30 \mathrm{mg} \cdot \mathrm{l}-1$ de agua de aceite esencial, es suficiente para la inducción anestésica (Azambuja et al., 2011), reduciendo así los daños que se producen por la movilidad de los peces.

Finalmente, la aplicación del aceite esencial de orégano ha demostrado ser eficaz inhibidor de la microbiota superficial de los filetes de merluza, especialmente cuando son incorporados en soluciones formadoras de films en concentraciones del $3 \%$ o superiores, incrementando la vida de anaquel de los filetes de peces. Por lo tanto, el uso del aceite 
esencial de orégano puede estar presente en el desarrollo de los sistemas intensivos de peces; debido a que como se mencionó anteriormente, su eficiencia abarca desde el desarrollo del crecimiento de los alevines, hasta el punto final del sistema productivo que es la conservación de los filetes en anaquel.

\section{CONCLUSIONES}

Los aceites esenciales de orégano de las subespecies vulgare y Lippia, son una alternativa como aditivos en la alimentación de los animales domésticos; mejoran la funcionalidad del sistema digestivo en monogástricos, reducen las emisiones de metano entérico en rumiantes y los malos olores en las heses de los cerdos. Las evidencias cientifícas demuestran que los aceites esenciales de orégano pueden ayudar a solucionar los problemas en los sistemas de producción animal.

\section{LITERATURA CITADA}

AANYU M, Betancor MB, Monroig O. 2018. Effects of dietary limonene and thymol on the growth and nutritional physiology of Nile tilapia (Oreochromis niloticus). Aquaculture. 488: 217-226.

ABDOLLAHZADEH E, Rezaei M, Hosseini H. 2014. Antibacterial activity of plant essential oils and extracts: The role of thyme essential oil, nisin, and their combination to control Listeria monocytogenes inoculated in minced fish meat. Food control. 35:177-183.

AIYEGORO O, Okoh A. 2009. Use of bioactive plant products in combination with standard antibiotics: implications in antimicrobial chemotherapy. Journal of Medicinal Plants Research. 3:1147-1152.

ALBADO PLAUS E, Saez Flores G, Grabiel Ataucusi S. 2001. Composición química y actividad antibacteriana del aceite esencial del Origanum vulgare (orégano). Revista Médica Herediana. 12, 16-19.

ALI B, Al-Wabel NA, Shams S, Ahamad A, Khan SA, Anwar F. 2015. Essential oils used in aromatherapy A systemic review. Asian Pacific Journal of Tropical Biomedicine. 5:601611.

ALIGIANNIS N, Kalpoutzakis E, Mitaku S, Chinou IB. 2001. Composition and antimicrobial activity of the essential oils of two Origanum species. Journal of agricultural and food chemistry. 49:4168-4170.

ALVAREZ JG, Storey BT. 1992. Evidence for increased lipid peroxidative damage and loss of superoxide dismutase activity as a mode of sublethal cryodamage to human sperm during cryopreservation. Journal of Andrology. 13:232-241.

ALLAN P, Bilkei G. 2005. Oregano improves reproductive performance of sows. Theriogenology. 63:716-721.

ARCILA-LOZANO CC, Loarca-Piña G, Lecona-Uribe S, González De Mejía E. 2004. El orégano: propiedades, composición y actividad biológica de sus componentes. Archivos Latinoamericanos de nutrición. 54:100-111. 
ARIZA-NIETO C, Bandrick M, Baidoo SK, Anil L, Molitor TW, Hathaway M. 2011. Effect of dietary supplementation of oregano essential oils to sows on colostrum and milk composition, growth pattern and immune status of suckling pigs. Journal of Animal Science. 89:1079-1089.

AZAMBUJA CR, Mattiazzi J, Riffel APK, Finamor IA, De Oliveira Garcia L, Heldwein CG, Heinzmann BM, Baldisserotto B, Pavanato MA, Llesuy SF. 2011. Effect of the essential oil of Lippia alba on oxidative stress parameters in silver catfish (Rhamdia quelen) subjected to transport. Aquaculture. 319:156-161.

BARRETO M, Menten JFM, Racanicci A, Pereira P, Rizzo P. 2008. Plant extracts used as growth promoters in broilers. Brazilian Journal of Poultry Science. 10:109-115.

BENCHAAR C, Chaves A, Fraser G, Yang Y, Beauchemin K, Mcallister T. 2007. Effects of essential oils and their components on $I n$ vitro rumen microbial fermentation. Can $J$ Anim Sci. 87:413-419.

BENCHAAR C, Greathead H. 2011. Essential oils and opportunities to mitigate enteric methane emissions from ruminants. Animal Feed Science and Technology. 166:338-355.

BOTSOGLOU N, Florou-Paneri P, Christaki E, Fletouris D, Spais A. 2002. Effect of dietary oregano essential oil on performance of chickens and on iron-induced lipid oxidation of breast, thigh and abdominal fat tissues. British poultry science. 43:223-230.

BOTSOGLOU N, Grigoropoulou S, Botsoglou E, Govaris A, Papageorgiou G. 2003. The effects of dietary oregano essential oil and a-tocopheryl acetate on lipid oxidation in raw and cooked turkey during refrigerated storage. Meat Science. 65:1193-1200.

BUSQUET M, Calsamiglia S, Ferret A, Kamel C. 2006. Plant Extracts Affect In Vitro Rumen Microbial Fermentation. Journal of Dairy Science. 89(2).

CABRERA CE, Gómez RF, Zúñiga AE. 2007. La Resistencia de Bacterias a Antibióticos, Antisépticos y Desinfectantes una Manifestación de los Mecanismos de Supervivencia y Adaptación. Colombia médica. 38:149-158.

CÁCERES RUEDA DE LEÓN, I, Colorado Vargas R, Salas Muñoz E, Muñoz Castellanos LN, Hernández Ochoa L. 2014. Actividad Antifúngica in vitro de Extractos Acuosos de Especias contra Fusarium oxysporum, Alternaría alternata, Geotrichum candidum, Trichoderma spp., Penicillum digitatum y Aspergillus niger. Revista mexicana de fitopatología. 31:105-112.

CASILLAS-ALCALÁ C. 1992. El Orégano en México: Panorama del Primer Exportador Mundial, ITESO. México.

CASTILLO IO, Almazán AJS, Arellano JDJE, Vázquez C. 2017. Recoleccion Y Comercializacion Del Oregano (Lippia spp) En El Semi-Desierto Mexicano, Un Caso De Estudio: Reserva Ecologica Municipal Sierra Y Cañon De Jimulco, Mexico. Revista Mexicana de Agronegocios. 41:684-695.

CAZARES ALONSO NP, Villavicencio Gutiérrez EE, Verde Sta J, Pecina Quintero V, León A, Humberto I. 2010. Caracterización molecular y producción de aceites esenciales de diferentes genotipos de orégano (Lippia spp). Revista mexicana de ciencias forestales. $1: 85-94$. 
CeRvato G, Carabelli M, Gervasio S, Cittera A, Cazzola R, Cestaro B. 2000. Antioxbdant properties of oregano (Origanum vulgare) leaf extracts. Journal of Food Biochemistry. 24:453-465.

CORONADO BASTIDAS MP. 2019. Efecto de niveles de inclusión de aceite esencial de orégano (Organum Vulgare ssp. hirtum) sobre parámetros productivos y económicos en post-larvas de Tilapia Roja (Oreochromis spp) en fase de mascuinización.

CORELLA-BERNAL RA, Ortega-Nieblas MM. 2013. Importancia del aceite esencial y la producción de orégano Lippia palmeri Watson en el estado de Sonora. Biotecnia. 15:5764. ISSN: 1665-1456.

COSTA C, Lodesani M, Maistrello L. 2010. Effect of thymol and resveratrol administered with candy or syrup on the development of Nosema ceranae and on the longevity of honeybees (Apis mellifera L.) in laboratory conditions. Apidologie. 41:141-150.

CONAFOR. 2009. Fichas de información comercial de productos forestales.

DOMÍNGUEZ-MARTÍNEZ P, Ávila-Ramos F, Carmona-Gasca C, Macías-Coronel H, Escalera-Valente F, Mario-Mendoza J. 2015. Effect of dietary oregano oil on the quantity of Aerobic Mesophilic detected in fresh and frozen broiler breast. Abanico Veterinario. 5(3):13-19.

DORMAN H, Peltoketo A, Hiltunen R, Tikkanen M. 2003. Characterisation of the antioxidant properties of de-odourised aqueous extracts from selected Lamiaceae herbs. Food chemistry. 83:255-262.

DURMIC Z, Moate PJ, Eckard R, Revell DK, Williams R, Vercoe PE. 2014. In vitro screening of selected feed additives, plant essential oils and plant extracts for rumen methane mitigation. Journal of the Science of Food and Agriculture. 94:1191-1196.

ESCALERA-VALENTE F, Loya-Olguín JL, Carmona-Gasca CA, Martínez-González S, Ávila-Ramos F. 2016. Effect of oregano oil supplementation in diets formed using either crude soybean oil or acidulated soybean oil soapstock as source of energy on the growth performance parameters of broilers. Archivos de Medicina Veterinaria. 48(2):209-214.

ELGAYYAR M, Draughon F, Golden D, Mount J. 2001. Antimicrobial activity of essential oils from plants against selected pathogenic and saprophytic microorganisms. Journal of food protection. 64:1019-1024.

FONNEGRA FG. 2007. Plantas medicinales aprobadas en Colombia, Universidad de Antioquia. ISBN:978-958-655-999-7;

FONSECA-GARCÍA I, Escalera-Valente F, Martínez-González S, Carmona-Gasca CA, Gutiérrez-Arenas DA, Ávila-Ramos F. 2017. Effect of oregano oil dietary supplementation on production parameters, height of intestinal villi and the antioxidant capacity in the breast of broiler. Austral journal of veterinary sciences. 49(2):83-89.

GARCÍA-CAMARILLO EA, Quezada-Viay MY, Moreno-Lara J, Sánchez-Hernández G, Moreno-Martínez E, Pérez-Reyes MCJ. 2006. Actividad antifúngica de aceites esenciales de canela (Cinnamomum zeylanicum Blume) y orégano (Origanum vulgare L.) y su efecto sobre la producción de aflatoxinas en nuez pecanera [Carya illinoensis (FA Wangenh) $\mathrm{K}$. Koch]. Revista Mexicana de Fitopatología. 24:8-12 
GARCÍA-PÉREZ E, Fernando Francisco CA, Gutiérrez-Uribe JA, García-Lara S. 2012. Revisión de la producción, composición fitoquímica y propiedades nutracéuticas del orégano mexicano. Revista mexicana de ciencias agrícolas. 3:339-353.

GERACI JI, Garciarena AD, Gagliostro GA, Beauchemin KA, Colombatto D. 2012. Plant extracts containing cinnamaldehyde, eugenol and capsicum oleoresin added to feedlot cattle diets: Ruminal environment, short term intake pattern and animal performance. Animal feed science and technology. 176:123-130.

GONÇALVES CATTELAN M, Bonatto Machado De Castilhos M, Juliana Pinsetta Sales P, Leite Hoffmann F. 2013. Antibacterial activity of oregano essential oil against foodborne pathogens. Nutrition and Food Science. 43:169-174.

GONZÁLEZ MH, Cortés ND, Centeno FH, Vázquez RS. 2009. Aplicación de aceite esencial de orégano mexicano (Lippia berlanderi Schauer) contra mesfilos aerobios y patógenos en carne de pavo. Nacameh. 3(2):48-56.

GOTSIOU P, Naxakis G, Skoula M. 2002. Diversity in the composition of monoterpenoids of Origanum microphyllum (Labiatae). Biochemical systematics and ecology. 30:865-879.

GOVARIS A, Solomakos N, Pexara A, Chatzopoulou P. 2010. The antimicrobial effect of oregano essential oil, nisin and their combination against Salmonella Enteritidis in minced sheep meat during refrigerated storage. International journal of food microbiology. 137: 175-180.

GÜERECA MCG, Hernández MS, Kite G, Vázquez MM. 2007. Actividad antioxidante de flavonoides del tallo de orégano mexicano (Lippia graveolens HBK var. berlandieri Schauer). Revista Fitotecnia Mexicana. 30:43-49.

GUERRA C, Galán JA, Méndez J, Perea EM. 2008. Evaluación del efecto del extracto de orégano (Oreganum vulgare) sobre algunos parámetros productivos de cerdos destetos. Tumbaga. 1(3):16-29.

HAZZIT M, Baaliouamer A, Faleiro ML, Miguel MG. 2006. Composition of the essential oils of Thymus and Origanum species from Algeria and their antioxidant and antimicrobial activities. Journal of agricultural and food chemistry. 54:6314-6321.

HERNANDEZ F, Madrid J, Garcia V, Orengo J, Megias M. 2004. Influence of two plant extracts on broilers performance, digestibility, and digestive organ size. Poultry science. 83:169-174.

HRISTOV AN, Lee C, Cassidy T, Heyler K, Tekippe JA, Varga GA, Brandt RC. 2013. Effect of Origanum vulgare $L$. leaves on rumen fermentation, production, and milk fatty acid composition in lactating dairy cows. Journal of Dairy Science. 96(2):1189-1202.

HUERTA C. 1997. Orégano mexicano: oro vegetal. Biodiversitas. 15:8-13.

HYLDGAARD M, Mygind T, Meyer RL. 2012. Essential oils in food preservation: mode of action, synergies, and interactions with food matrix components. Frontiers in microbiology. 3:12.

INFOAGRO 2006. El cultivo de orégano. Disponible en: https://www.infoagro.com/aromaticas/oregano.htm 
ISABEL B, Santos Y. 2009. Efectos de los aceites esenciales en la alimentación de los pollos de carne. Archivos de Zootecnia. 58:597-600.

ITZÁ WDJM, Medina LAM, Olivares JCM. 2007. Eficacia de un gel a base de timol en el control del ácaro Varroa destructor que infesta colonias de abejas Apis mellifera, bajo condiciones tropicales en Yucatán, México. Veterinaria México. 38:1-8.

KLEVENHUSEN F, Zeitz J, Duval S, Kreuzer M, Soliva C. 2011. Garlic oil and its principal component diallyl disulfide fail to mitigate methane, but improve digestibility in sheep. Animal Feed Science and Technology. 166:356- 363.

KHAJARERN, J. Y Khajarern, S. 2002. La eficacia de los aceites esenciales de origanum en la alimentación de cerdas. Int Pig Topics. 17(17).

KNIGHT J. 2010. Invasive ornamental fish: a potential threat to aquatic biodiversity in peninsular India. Journal of Threatened Taxa. 2(2):700-704.

KOKSAL O, Gunes E, Ozer OO, Ozden M. 2010. Analysis of effective factors on information sources at Turkish Oregano farms. African Journal of Agricultural Research. 5:142-149.

KULISIC T, Radonic A, Katalinic V, Milos M. 2004. Use of different methods for testing antioxidative activity of oregano essential oil. Food chemistry. 85:633-640.

LOEZA-CONCHA H, Domínguez-Rebolledo A, Escalera-Valente F, Ávila-Ramos F, Carmona-Gasca C. 2018. Identificación morfométrica de Varroa destructor y su plasticidad por la exposición a timol. Abanico veterinario. 8:98-107.

MARTíNEZ SANTIAGO A, Vázquez Silva G, Martínez García J, Arana Magallón F, Núñez García L, López De La Rosa A, Rodríguez Vicente A. 2018. Aceite Esencial de Orégano Mexicano como Antimicrobiano de Bacterias Aisladas de Peces de Xochimilco: una Alternativa en la Producción Acuícola. Avances de la Investigación Sobre Producción Animal y Seguridad Alimentaria en México. Pp.1003-1008. ISBN: 978-607-542-022-6.

MADSEN HL, Bertelsen G. 1995. Spices as antioxidants. Trends in food science and technology. 6:271-277.

MAISTRELLO L, Lodesani M, Costa C, Leonardi F, Marani G, Caldon M, Mutinelli F, Granato A. 2008. Screening of natural compounds for the control of nosema disease in honeybees (Apis mellifera). Apidologie. 39:436-445.

MARTíNEZ R, Cerrilla M, Haro J, Garza J, Ramos J, Soriano R. 2015. Uso de aceites esenciales en animales de granja. Interciencia. 40:744-750.

MENESES R, Ocazionez RE, Martínez JR, Stashenko EE. 2009. Inhibitory effect of essential oils obtained from plants grown in Colombia on yellow fever virus replication in vitro. Annals of Clinical Microbiology and Antimicrobials. 8(8).

MOREIRAS O, Carbajal A, Cabrera L, Cuadrado C. 2013. Tabla de composición de alimentos. $\quad 16 \quad$ edición. $\quad$ Madrid: $\quad$ Ed. Pirámide. http://www.sennutricion.org/es/2013/05/14/tablas-de-composicin-de-alimentos-moreiraset-al 
MONU EA, Techathuvanan C, Wallis A, Critzer FJ, Davidson PM. 2016. Plant essential oils and components on growth of spoilage yeasts in microbiological media and a model salad dressing. Food control. 65:73-77.

National Library of Medicine. Oreganum. Consultado el 14 de noviembre de 2019. Disponible en: https://www.ncbi.nlm.nih.gov/Taxonomy/Browser/wwwtax.cgi?id=39174

NEWBOLD CJ, Mcintosh Fm, Williams P, Losa R, Wallace R. 2004 Effects of a spesific blend of essential oil compounds on rumen fermentation. Anim Feed Sci Tech. 114:105112.

NIEVES CG, Ávila JA, Herrera GG, Lopez JR, Flores RC, Arriaga OE, Castorena MV. 2010. Efectos de Tratamientos Pregerminativos en la Emergencia y Crecimiento de Plántulas de Orégano (Lippia graveolens HBK). Revista Chapingo Serie Zonas Áridas. 9: 129-134.

OETTING LL, Utiyama CE, Giani PA, Ruiz UDS, Miyada VS. 2006. Efectos de los extractos de plantas y antimicrobianos sobre la digestibilidad aparente, el rendimiento, la morfometría de los órganos y la histología intestinal de lechones recién destetados. Revista Brasileña de Zootecnia. 35(4):1389-1397.

PERALTA JIMENEZ YJ. 2018. Evaluación de la actividad antiinflamatoria del extracto etanólico de Origanum vulgare L." Orégano" en ratas albinas. Tesis de Licenciatura. Universidad San Pedro. Facultad de Medicina Humana. Perú.

RICE RN. 2001. Nosema Disease in Honeybees: Genetic Variation and Control: a Report for the Rural Industries Research and Development Corporation. RIRDC. ISBN: 642 582688.

ROMO-CHACÓN A, Martínez-Contreras LJ, Molina-Corral FJ, Acosta-Muñiz CH, RíosVelasco C, De León-Door AP, Rivera R. 2016. Evaluation of Oregano (Lippia berlandieri) Essential Oil and Entomopathogenic Fungi for Varroa destructor Control in Colonies of Honey Bee, Apis mellifera. Southwestern Entomologist. 41(4):971-983.

SANTOYO S, Cavero S, Jaime L, Ibanez E, Senorans F, Reglero G. 2006. Supercritical carbon dioxide extraction of compounds with antimicrobial activity from Origanum vulgare L. Determination of optimal extraction parameters. Journal of food protection. 69:369-375.

SOTO-DOMíNGUEZ A, García-Garza R, Ramírez-Casas Y, Morán-Martínez J, SerranoGallardo LB. 2012. El extracto acuoso de orégano (Lippia graveolens HBK) del norte de México tiene actividad antioxidante sin mostrar un efecto toxico in vitro e in vivo. International Journal of Morphology. 30:937-944.

SOTO RJ, Hernández AF, Franco RC, Silva R. 2007. Identificación y selección de genotipos de orégano (Lippia berlandieri Schauer) sobresalientes en producción de timol y carvacrol. Revista Chapingo Serie Zonas Áridas. 6:25-36.

TALEBZADEH R, Alipour D, Saharkhiz M, Azarfar A, Malecky M. 2012. Effect of essential oils of Zataria multiflora on in vitro rumen fermentation, protozoal population, growth and enzyme activity of anaerobic fungus isolated from Mehraban sheep. Animal feed science and technology. 172:115-124. 
VAN DEN HEEVER JP, Thompson TS, Otto SJ, Curtis JM, Ibrahim A, Pernal SF. 2016. Evaluation of Fumagilin- $\mathrm{B} B$ and other potential alternative chemotherapies against Nosema ceranae-infected honeybees (Apis mellifera) in cage trial assays. Apidologie. 47: 617-630.

VAREL VH. 2002. Carvacrol and thymol reduce swine waste odor and pathogens: stability of oils. Current microbiology. 44:38-43.

VIUDA-MARTOS M, Mohamady M, Fernández-López J, Elrazik KA, Omer E, PérezAlvarez J, Sendra E. 2011. In vitro antioxidant and antibacterial activities of essentials oils obtained from Egyptian aromatic plants. Food Control. 22:1715-1722.

World checklist of selected plant families (WCSP). Royal Botanic Gardens, Kew Science. London, England.

YANISHLIEVA NV, Marinova EM, Gordon MH, Raneva VG. 1999. Antioxidant activity and mechanism of action of thymol and carvacrol in two lipid systems. Food Chemistry. 64:5966.

YANO Y, Satomi M, Oikawa H. 2006. Antimicrobial effect of spices and herbs on Vibrio parahaemolyticus. International Journal of Food Microbiology. 111:6-11.

ZOU Y, Xiang Q, Wang J, Peng J, Wei H. 2016. Oregano essential oil improves intestinal morphology and expression of tight junction proteins associated with modulation of selected intestinal bacteria and immune status in a pig model. BioMed research international. 2016:1-11. 